\title{
Identifying Dynamic Games with Serially-Correlated Unobservables*
}

\author{
Yingyao $\mathrm{Hu}$ \\ Dept. of Economics, Johns Hopkins University \\ Matthew Shum \\ Division of Humanities and Social Sciences, Caltech \\ First draft: August 20, 2008 \\ This draft: October 13, 2008 \\ Preliminary: \\ Comments welcome
}

\begin{abstract}
In this paper we consider the nonparametric identification of Markov dynamic games models in which each firm has its own unobserved state variable, which is persistent over time. This class of models includes most models in the Ericson and Pakes (1995) and Pakes and McGuire (1994) framework. We provide conditions under which the joint Markov equilibrium process of the firms' observed and unobserved variables can be nonparametrically identified from data. For stationary continuous action games, we show that only three observations of the observed component are required to identify the equilibrium Markov process of the dynamic game. When agents' choice variables are discrete, but the unobserved state variables are continuous, four observations are required.
\end{abstract}

\section{Introduction}

In this paper, we consider nonparametric identification in Markovian dynamic games models where which each agent may have its own serially-correlated unobserved state variable. This class of models includes most models in the Ericson and Pakes (1995) and Pakes and McGuire (1994) framework. These models have been the basis for much of the recent

${ }^{*}$ The authors can be reached at yhu@jhu. edu and mshum@caltech.edu. 
empirical applications of dynamic game models. Throughout, by "unobservable", we mean variables which are commonly observed by all agents, and condition their actions, but are unobserved by the researcher.

Consider a dynamic duopoly game in which two firms compete. It is straightforward to extend our assumptions and arguments to the case of $N$ firms. A dynamic duopoly is described by the sequence of variables $\left(W_{t+1}, X_{t+1}^{*}\right),\left(W_{t}, X_{t}^{*}\right), \ldots,\left(W_{1}, X_{1}^{*}\right)$ where

$$
\begin{aligned}
& W_{t}=\left(W_{1, t}, W_{2, t}\right), \\
& X_{t}^{*}=\left(X_{1, t}^{*}, X_{2, t}^{*}\right) .
\end{aligned}
$$

$W_{i, t}$ stands for the observed information on firm $i$ and $X_{i, t}^{*}$ denote the unobserved heterogeneity of firm $i$ at period $t$, which we allow to vary over time and be serially-correlated.

In empirical dynamic games model, the observed variables $W_{i, t}$ consists of two variables:

$$
W_{i, t} \equiv\left(Y_{i, t}, M_{i, t}\right)
$$

where $Y_{i, t}$ denotes firm $i$ 's choice, or control variable in period $t$, and $M_{i, t}$ denotes the state variables of firm $i$ which are observed by both the firms and the researcher. We assume that the serially-correlated variables $X_{1, t}^{*}$ and $X_{2, t}^{*}$ are observed by both firms prior to making their choices of $Y_{1, t}, Y_{2, t}$ in period $t$, but the researcher never observes $X_{t}^{*}$. For simplicity, we assume that each firm's variables $Y_{i, t}, M_{i, t}, X_{i, t}^{*}$ are scalar-valued.

Main Results: Our goal is to identify the density

$$
f_{W_{t}, X_{t}^{*} \mid W_{t-1}, X_{t-1}^{*}},
$$

which corresponds to the equilibrium transition density of the choice and state variables along the Markov equilibrium path of the dynamic game. In Markovian dynamic settings, the transition density can be factored into two components of interest:

$$
\begin{aligned}
f_{W_{t}, X_{t}^{*} \mid W_{t-1}, X_{t-1}^{*}} & =f_{Y_{t}, M_{t}, X_{t}^{*} \mid Y_{t-1}, M_{t-1}, X_{t-1}^{*}} \\
& =\underbrace{f_{Y_{t} \mid M_{t}, X_{t}^{*}}}_{\mathrm{CCP}} \cdot \underbrace{f_{M_{t}, X_{t}^{*} \mid Y_{t-1}, M_{t-1}, X_{t-1}^{*}}^{*}}_{\text {state transition }} .
\end{aligned}
$$


The first term denotes the conditional choice probabilities (CCP) for the firms' actions in period $t$, conditional on the current state $\left(M_{t}, X_{t}^{*}\right)$. In the Markov equilibrium, firms' optimal strategies typically depends just on the current state variables $\left(M_{t}, X_{t}^{*}\right)$, but not past values. The second term denotes is the equilibrium Markovian transition probabilities for the state variables $\left(M_{t}, X_{t}^{*}\right)$. As shown in Hotz and Miller (1993) and Magnac and Thesmar (2002), once these two structural components are known, it is possible to recover the "deep" structural elements of the model, including the period utility functions.

In an earlier paper (Hu and Shum (2008)), we focused on nonparametric identification of Markovian single-agent dynamic optimization models. There, we showed that in stationary models, four periods of data $W_{t+1}, \ldots, W_{t-2}$ were enough to identify the Markov transition $W_{t}, X_{t}^{*} \mid W_{t-1}, X_{t-1}^{*}$, while five observations $W_{t+1}, \ldots, W_{t-3}$ were required for the nonstationary case. In this paper, we focus on Markovian dynamic games. We show that, once additional features of the dynamic optimization framework are taken into account, only three observations $W_{t}, \ldots, W_{t-2}$ are required to identify $W_{t}, X_{t}^{*} \mid W_{t-1}, X_{t-1}^{*}$ in the stationary case, when $Y_{t}$ is a continuous choice variable. If $Y_{t}$ is a discrete choice variable (while $X_{t}^{*}$ is continuous), then four observations are required for identification.

Related literature Recently, there has been a growing literature related to identification and estimation of dynamic games. Papers include Aguirregabiria and Mira (2007), Pesendorfer and Schmidt-Dengler (2007), Bajari, Benkard, and Levin (2007), Pakes, Ostrovsky, and Berry (2007), and Bajari, Chernozhukov, Hong, and Nekipelov (2007). Our main contribution relative to this literature is to provide nonparametric identification results for the case where there are firm-specific unobserved state variables, which are serially correlated over time. Allowing for firm-specific and serially-correlated unobservables is important, because the dynamic game models in Ericson and Pakes (1995) and Pakes and McGuire (1994) (see also Doraszelski and Pakes (2007)), which provide an important framework for much of the existing empirical work in dynamic games, explicitly contain firm-specific "product quality" variables which are typically unobserved by researchers.

A few recent papers have considered estimation methodologies for games with seriallycorrelated unobservables. Arcidiacono and Miller (2006) develop an EM-algorithm for estimating dynamic games where the unobservables are assumed to follow a discrete Markov process. Siebert and Zulehner (2008) extend the Bajari, Benkard, and Levin (2007) approach to estimate a dynamic product choice game for the computer memory industry where each firm experiences a serially-correlated productivity shock. Finally, Blevins (2008) de- 
velops simulation estimators for dynamic games with serially-correlated unobservables, utilizing state-of-the-art recursive importance sampling ("particle filtering") techniques. However, all these papers focus on estimation of parametric models in which the parameters are assumed to be identified, whereas this paper concerns nonparametric identification.

The class of models considered in this paper also resemble models analyzed in the dynamic treatment effects literature in labor economics (eg. Cunha, Heckman, and Schennach (2006), Abbring and Heckman (2007), Heckman and Navarro (2007)). Specifically, Cunha, Heckman, and Schennach (2006) apply the result of Hu and Schennach (2008) to show nonparametric identification of a nonlinear factor model consisting of $\left(W_{t}, W_{t}^{\prime}, W_{t}^{\prime \prime}, X_{t}^{*}\right)$, where the observed processes $\left\{W_{t}\right\}_{t=1}^{T},\left\{W_{t}^{\prime}\right\}_{t=1}^{T}$, and $\left\{W_{t}^{\prime \prime}\right\}_{t=1}^{T}$ constitute noisy measurements of the latent process $\left\{X_{t}^{*}\right\}_{t=1}^{T}$, contaminated with random disturbances. In contrast, we consider a different setting where $\left(W_{t}, X_{t}^{*}\right)$ jointly evolves as a dynamic first-order Markov process.

\section{Examples of Dynamic Duopoly Games}

To make things concrete, we present two examples of a dynamic duopoly problem, both of which are in the "dynamic investment" framework of Ericson and Pakes (1995) and Pakes and McGuire (1994), but simplified without an entry decision.

Example 1 is a model of learning by doing in a durable goods market, similar to Benkard (2004). There are two heterogeneous firms $i=1,2$, with each firm described by two timevarying state variables $\left(M_{i, t}, X_{i, t}^{*}\right) . M_{i, t}$ denotes the "installed base" of firm $i$, which are the share of consumers who have previously bought firm $i$ 's product. $X_{i, t}^{*}$ is firm $i$ 's marginal cost, which is unobserved to the econometrician, and is an unobserved state variable. There is learning by doing, in the sense that increases in the installed base will lower future marginal costs. In each period, each firm's choice variable $Y_{i, t}$ is its period $t$ price, which affects the demand for its product in period $t$ and thereby the future installed base, which in turn affects future production costs.

In the following, let $Y_{t} \equiv\left(Y_{1, t}, Y_{2, t}\right)$, and similarly for $M_{t}$ and $X_{t}^{*}$. Let $S_{t} \equiv\left(M_{t}, X_{t}^{*}\right)$ denote the payoff-relevant state variables of the game in period $t$. $S_{i, t} \equiv\left(M_{i, t}, X_{i, t}^{*}\right)$, for $i=1,2$, denotes firm $i$ 's state variables. Each period, firms earn profits by selling their products to 
consumers who have not yet bought the product. The demand curve for firm $i$ 's product is

$$
q_{i}\left(Y_{t}, M_{t}, \eta_{i, t}\right)
$$

which depends on the price and installed base of both firms' products. Firm $i$ 's demand also depends on $\eta_{i, t}$, a firm-specific demand shock. As in Aguirregabiria and Mira (2007) and Pesendorfer and Schmidt-Dengler (2007), we assume that $\eta_{i, t}$ is privately observed by each firm; that is, only firm 1 , but not firm 2 , observes $\eta_{1, t}$, making this a game of incomplete information. Furthermore, we assume that the demand shocks $\eta_{i, t}$ are i.i.d. across firm and periods, and distributed according to a distribution $K$ which is common knowledge to both firms. The main role of the variable $\eta_{i, t}$ is to generate randomness in $Y_{i, t}$, even after conditioning on $\left(M_{t}, X_{t}^{*}\right)$.

The period $t$ profits of firm $i$ can then be written:

$$
\Pi_{i}\left(Y_{t}, S_{t}, \eta_{i, t}\right)=q_{i}\left(Y_{t}, M_{t}, \eta_{i, t}\right) *\left(Y_{i, t}-X_{i, t}^{*}\right)
$$

where $Y_{i, t}-X_{i, t}^{*}$ is firm $i$ 's margin from each unit that it sells.

Installed base evolves according to the conditional distribution:

$$
M_{i, t+1} \sim G\left(\cdot \mid M_{i, t}, Y_{i, t}\right) .
$$

One example is to model the incremental change $M_{i, t+1}-M_{i, t}$ as a log-normal random variable

$$
\log \left(M_{i, t+1}-M_{i, t}\right) \sim q_{i}\left(Y_{t}, M_{t}, \eta_{i, t}\right)+\epsilon_{i, t}, \quad \epsilon_{i, t} \sim N\left(0, \sigma_{\epsilon}^{2}\right) \text {, i.i.d.- }(i, t)
$$

Marginal cost evolves according to the conditional distribution

$$
X_{i, t+1}^{*} \sim H\left(\cdot \mid X_{i, t}^{*}, M_{i, t+1}\right)
$$

One example is

$$
X_{i, t+1}^{*}=X_{i, t}^{*}-N\left(\gamma\left(M_{i, t+1}-M_{i, t}\right), \sigma_{k}^{2}\right)
$$

where $\gamma$ and $\sigma_{k}$ are unknown parameters. This encompasses learning-by-doing because the incremental reduction in marginal cost $\left(X_{i, t+1}^{*}-X_{i, t}^{*}\right)$ depends on the incremental increase 
in installed base $\left(M_{i, t+1}-M_{i, t}\right)$.

In the dynamic Markov-perfect equilibrium, each firm's optimal pricing strategy will also be a function of the current $S_{t}$, and the current demand shock $\eta_{i, t}$ :

$$
Y_{i, t}=Y_{i}^{*}\left(S_{t}, \eta_{i, t}\right), \quad i=1,2
$$

where the strategy satisfies the equilibrium Bellman equation:

$$
\begin{gathered}
Y_{i}^{*}\left(S_{t}, \eta_{i, t}\right)=\operatorname{argmax}_{y} E_{\eta_{-i, t}}\left\{\Pi_{i}\left(S_{t}, y, Y_{-i, t}=Y_{-i}^{*}\left(S_{t}, \eta_{-i, t}\right)\right)+\right. \\
\left.\beta E\left[V_{i}\left(S_{t+1}, \eta_{i, t+1}\right) \mid y, Y_{-i, t}=Y_{-i}^{*}\left(S_{t}, \eta_{-i, t}\right)\right]\right\}
\end{gathered}
$$

subject to Eqs. (4) and (3). In the above equation, $V_{i}\left(S_{t}, \eta_{i t}\right)$ denotes the equilibrium value function for firm $i$, which is equal to the expected discounted future profits that firm $i$ will make along the equilibrium path, starting at the current state $\left(S_{t}, \eta_{i t}\right)$.

Example 2 is a simplified version of the dynamic investment models estimated in the productivity literature. (See Ackerberg, Benkard, Berry, and Pakes (2007) for a detailed survey of this literature.) In this model, firms' state variables are $\left(M_{i, t}, X_{i, t}^{*}\right)$, where $M_{i, t}$ denotes firm $i$ 's capital stock, and $X_{i, t}^{*}$ denotes its productivity shock in period $t . Y_{i, t}$, firm $i$ 's choice variable, denotes new capital investment in period $t$.

Capital stock $M_{i, t}$ evolves deterministically, as a function of $\left(Y_{i, t-1}, M_{i, t-1}\right)$ :

$$
M_{i, t}=(1-\delta) \cdot M_{i, t-1}+Y_{i, t-1}
$$

The productivity shock is serially correlated, and evolves according to the conditional distribution

$$
X_{i, t+1}^{*} \sim H\left(\cdot \mid X_{i, t}^{*}, M_{i, t}\right)
$$

Each period, firms earn profits by selling their products. Let $q_{i}\left(p_{i, t}, p_{-i, t}, \eta_{i, t}\right)$ denote the demand curve for firm $i$ 's product, which depends on the quality and prices of both firms' products. As in Example 1, $\eta_{i, t}$ denotes the privately observed demand shock for firm $i$ in period $t$, which is distributed i.i.d. across firms and time periods. 
The period $t$ profits of firm $i$ are:

$$
q_{i}\left(p_{i, t}, p_{-i, t}, \eta_{i, t}\right) *\left(p_{i, t}-c_{i}\left(S_{i, t}\right)\right)-K\left(Y_{i, t}\right)
$$

where $c_{i}(\cdot)$ is the marginal cost function for firm $i$ (we assume constant marginal costs) and $K\left(Y_{i t}\right)$ is the investment cost function.

Following the literature, we assume that each firm's price in period $t$ are determined by a static equilibrium, given the current values of the state variables $S_{t}$, and the firm-specific demand shock $\eta_{i, t}$. Let $p_{i}^{*}\left(S_{t}, \eta_{i, t}\right)$ denote the static equilibrium prices for each firm in period $t$. By substituting in the equilibrium prices in firm's profit function, we obtain each firm's "reduced-form" expected profits:

$\Pi_{i}\left(S_{t}, Y_{t}, \eta_{i, t}\right)=E_{\eta_{-i, t}} q_{i}\left(p_{1}^{*}\left(S_{t}, \eta_{1, t}\right), p_{2}^{*}\left(S_{t}, \eta_{2, t}\right), \eta_{i, t}\right) *\left[p_{i}^{*}\left(S_{t}, \eta_{i, t}\right)-c_{i}\left(S_{i, t}\right)\right]-K\left(Y_{i, t}\right), \quad i=1,2$

As in Example 1, the Markov equilibrium investment strategy for each firm just depends on the current state variables $S_{t}$, and the current shock $\eta_{i, t}$ :

$$
Y_{t}=Y_{i}^{*}\left(S_{t}, \eta_{i t}\right), \quad i=1,2
$$

subject to the Bellman equation (6) and the transitions (7) and (8).

The substantial difference between examples 1 and 2 is that in example 2, the evolution of the observed state variable $M_{i, t}$ is deterministic, whereas in example 1 there is randomness in $M_{i, t}$ conditional on $\left(M_{i, t-1}, Y_{i, t-1}\right)$ (i.e., compare Eqs. (3) and (7)). As we will see below, this has important implications for nonparametric identification.

Moreover, as illustrated in these two examples, for the first part of the paper we focus on games with continuous actions, so that $Y_{t}$ are continuous variables. Later, we will consider the important alternative case of discrete-action games, where $Y_{t}$ is discrete-valued.

\section{Nonparametric identification}

In this section, we present the assumptions for nonparametric identification in the dynamic game model. The assumption we make here are different than those in our earlier paper (Hu and Shum (2008)), and are geared specifically for the dynamic games literature, and 
motivated directly by existing applied work utilizing dynamic games. We assume that for each market $j,\left\{\left(W_{t+1}, X_{t+1}^{*}\right),\left(W_{t}, X_{t}^{*}\right), \ldots,\left(W_{1}, X_{1}^{*}\right)\right\}_{j}$ is an independent random draw

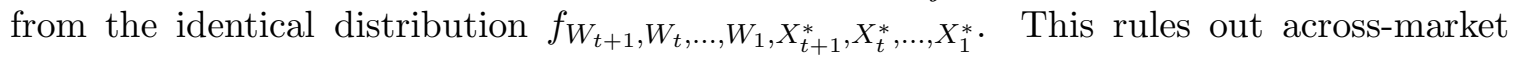
effects and spillovers. For each market $j,\left\{W_{1}, \ldots, W_{T}\right\}_{j}$ is observed, for $T \geq 4$.

After presenting each assumption, we relate it to the examples in the previous section. Define $\Omega_{<t}=\left\{W_{t-1}, \ldots, W_{1}, X_{t-1}^{*}, \ldots, X_{1}^{*}\right\}$. We assume the dynamic process satisfies:

Assumption 1 First-order Markov:

$$
f_{W_{t}, X_{t}^{*} \mid W_{t-1}, X_{t-1}^{*}, \Omega_{<t-1}}=f_{W_{t}, X_{t}^{*} \mid W_{t-1}, X_{t-1}^{*}}
$$

Remark: The first-order Markov assumption is satisfied along the Markov-equilibrium path of both examples given in the previous section.

Without loss of generality, we assume that $W_{t}=\left(Y_{t}, M_{t}\right) \in \mathbb{R}^{2}$. We assume

\section{Assumption 2}

$$
\begin{aligned}
& \text { (i) } f_{Y_{t} \mid M_{t}, X_{t}^{*}, Y_{t-1}, M_{t-1}, X_{t-1}^{*}}=f_{Y_{t} \mid M_{t}, X_{t}^{*}}, \\
& \text { (ii) } f_{X_{t}^{*} \mid M_{t}, Y_{t-1}, M_{t-1}, X_{t-1}^{*}}=f_{X_{t}^{*} \mid M_{t}, M_{t-1}, X_{t-1}^{*}} \text {. }
\end{aligned}
$$

Assumption 2(i) is motivated completely by the state-contingent aspect of the optimal policy function in dynamic optimization models. It turns out that this assumption is stronger than necessary for our identification, but it allows us to achieve identification only using three periods of data. Assumption 2(ii) implies that $X_{t}^{*}$ is independent of $Y_{t-1}$ conditional on $M_{t}, M_{t-1}$ and $X_{t-1}^{*}$. This is consistent with the setup above.

Remarks: Assumption 2 is satisfied in both examples 1 and 2 .

The conditional independence assumptions 1-2 imply that the Markov transition density (1) can be factored into

$$
\begin{aligned}
f_{W_{t}, X_{t}^{*} \mid W_{t-1}, X_{t-1}^{*}} & =f_{Y_{t}, M_{t}, X_{t}^{*} \mid Y_{t-1}, M_{t-1}, X_{t-1}^{*}} \\
& =f_{Y_{t} \mid M_{t}, X_{t}^{*}} \cdot f_{X_{t}^{*} \mid M_{t}, M_{t-1}, X_{t-1}^{*}} \cdot f_{M_{t} \mid Y_{t-1}, M_{t-1}, X_{t-1}^{*}} .
\end{aligned}
$$

In the identification procedure, we will identify these three components of $f_{W_{t}, X_{t}^{*} \mid W_{t-1}, X_{t-1}^{*}}$ 
in turn.

Next, we restrict attention to stationary equilibria in the dynamic game, which is natural given our focus on Markov equilibria. In stationary equilibria, the Markov transition density $f_{W_{t}, X_{t}^{*} \mid W_{t-1}, X_{t-1}^{*}}$ is time-invariant.

Assumption 3 Stationarity of Markov kernel:

$$
f_{W_{t}, X_{t}^{*} \mid W_{t-1}, X_{t-1}^{*}}=f_{W_{2}, X_{2}^{*} \mid W_{1}, X_{1}^{*}}
$$

Consider the joint density of $\left\{Y_{t}, M_{t}, Y_{t-1}, M_{t-1}, Y_{t-2}\right\}$. Let $\operatorname{supp}\left(Y_{t}, M_{t}, Y_{t-1}, M_{t-1}, Y_{t-2}\right)$ denote for the support of the random vector $\left\{Y_{t}, M_{t}, Y_{t-1}, M_{t-1}, Y_{t-2}\right\}$, together with $\mathcal{Y}_{t}=$ $\operatorname{supp}\left(Y_{t}\right), \mathcal{M}_{t}=\operatorname{supp}\left(M_{t}\right)$, and $\mathcal{X}_{t}^{*}=\operatorname{supp}\left(X_{t}^{*}\right)$.

We show in the Appendix, that Assumptions 1-2 imply that

$$
\begin{aligned}
& f_{Y_{t}, M_{t}, Y_{t-1} \mid M_{t-1}, Y_{t-2}} \\
= & \int f_{Y_{t} \mid M_{t}, M_{t-1}, X_{t-1}^{*}} f_{M_{t}, Y_{t-1} \mid M_{t-1}, X_{t-1}^{*}} f_{X_{t-1}^{*} \mid M_{t-1}, Y_{t-2}} d x_{t-1}^{*} \\
= & \int f_{Y_{t} \mid M_{t}, M_{t-1}, X_{t-1}^{*}} f_{M_{t}, Y_{t-1}, X_{t-1}^{*} \mid M_{t-1}, Y_{t-2}} d x_{t-1}^{*}
\end{aligned}
$$

where the final line follows from Assumptions 1-2.

Let $\omega(\cdot)$ be a user-specified function. For example, $\omega(\cdot)$ may be $\omega(x)=x, x^{2}, c, I(x>c)$, or $I(x=c)$ for some constant $c$. In practice, the choice of $\omega(\cdot)$ will be model-specific. As will be clear below, one reason we introduce the $\omega(\cdot)$ function is in order to accommodate games where firms' choice variables $Y_{i t}$ are deterministic functions of the current state $\left(M_{t}, X_{t}^{*}\right)$. (For both examples in the previous section, this would correspond to the case without the firm-specific shocks $\eta_{i t}$.) Given Eq. (11), it follows that, for any $\left(x, m_{t}, m_{t-1}, z\right) \in$ $\operatorname{supp}\left(Y_{t}, M_{t}, M_{t-1}, Y_{t-2}\right)$

$$
\begin{aligned}
& \int \omega\left(y_{t-1}\right) f_{Y_{t}, M_{t}, Y_{t-1} \mid M_{t-1}, Y_{t-2}}\left(x, m_{t}, y_{t-1} \mid m_{t-1}, z\right) d y_{t-1} \\
= & \int\left[f_{Y_{t} \mid M_{t}, M_{t-1}, X_{t-1}^{*}}\left(x \mid m_{t}, m_{t-1}, x_{t-1}^{*}\right) E\left[\omega\left(Y_{t-1}\right) \mid m_{t}, m_{t-1}, x_{t-1}^{*}\right] .\right. \\
& \left.f_{M_{t}, \mid M_{t-1}, X_{t-1}^{*}}\left(m_{t}, \mid m_{t-1}, x_{t-1}^{*}\right) f_{X_{t-1}^{*} \mid M_{t-1}, Y_{t-2}}\left(x_{t-1}^{*} \mid m_{t-1}, z\right)\right] d x_{t-1}^{*},
\end{aligned}
$$


where we use the shorthand notation

$$
E\left[\omega\left(Y_{t-1}\right) \mid m_{t}, m_{t-1}, x_{t-1}^{*}\right]=E\left[\omega\left(Y_{t-1}\right) \mid M_{t}=m_{t}, M_{t-1}=m_{t-1}, X_{t-1}^{*}=x_{t-1}^{*}\right] .
$$

In order to identify the unknown densities on the right hand side, we use the identification strategy for the nonclassical measurement error models in Hu and Schennach (2008). Their results imply that two measurements and a dependent variable of a latent explanatory variable are enough to achieve identification. For fixed values of $\left(M_{t}, M_{t-1}\right)$, we see that $\left(Y_{t}, Y_{t-1}, Y_{t-2}\right)$ enter equation (11) separately in, respectively, the first, second, and third terms. This implies that we can use $\left(Y_{t}, Y_{t-2}\right)$ as the two measurements and $Y_{t-1}$ as the dependent variable of the latent variable $X_{t-1}^{*}$.

Since the function $\omega(\cdot)$ is user-specified, the density on the left hand side of equation (12) is observed in the data. Let $\mathcal{L}^{p}(\mathcal{X}), 1 \leq p<\infty$ stand for the space of functions $h(\cdot)$ with $\int_{\mathcal{X}}|h(x)|^{p} d x<\infty$, and let $\mathcal{L}^{\infty}(\mathcal{X})$ denote the space of functions $h(\cdot)$ with $\sup _{x \in \mathcal{X}}|h(x)|<$ $\infty$. For any $1 \leq p \leq \infty$, We define the integral operator $L_{Y_{t}, m_{t}, \omega \mid m_{t-1}, Y_{t-2}}: \mathcal{L}^{p}\left(\mathcal{Y}_{t-2}\right) \rightarrow$ $\left.\mathcal{L}^{p}\left(\mathcal{Y}_{t}\right)\right)$ for any given $\left(m_{t}, m_{t-1}\right) \in \operatorname{supp}\left(M_{t}, M_{t-1}\right)$ and any $h \in \mathcal{L}^{p}\left(\mathcal{Y}_{t-2}\right)$,

$$
\begin{aligned}
\left(L_{Y_{t}, m_{t}, \omega \mid m_{t-1}, Y_{t-2}} h\right)(x) & =\int k\left(x, m_{t} \mid m_{t-1}, z\right) h(z) d z \\
k\left(x, m_{t} \mid m_{t-1}, z\right) & =\int \omega\left(y_{t-1}\right) f_{Y_{t}, M_{t}, Y_{t-1} \mid M_{t-1}, Y_{t-2}}\left(x, m_{t}, y_{t-1} \mid m_{t-1}, z\right) d y_{t-1} .
\end{aligned}
$$

We also define the operator corresponding to the unobserved density $f_{Y_{t} \mid M_{t}, M_{t-1}, X_{t-1}^{*}}$, i.e.,

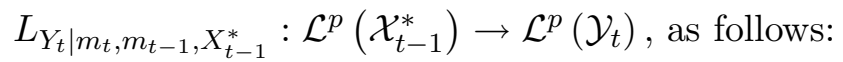

$$
\left(L_{Y_{t} \mid m_{t}, m_{t-1}, X_{t-1}^{*}} h\right)(x)=\int f_{Y_{t} \mid M_{t}, M_{t-1}, X_{t-1}^{*}}\left(x \mid m_{t}, m_{t-1}, x_{t-1}^{*}\right) h\left(x_{t-1}^{*}\right) d x_{t-1}^{*} .
$$

As shown in Hu and Schennach (2008), the identification of an operator, e.g., $L_{Y_{t} \mid m_{t}, m_{t-1}, X_{t-1}^{*}}$, is equivalent to that of its corresponding density, e.g., $f_{Y_{t} \mid M_{t}, M_{t-1}, X_{t-1}^{*}}$. Identification of $L_{Y_{t} \mid m_{t}, m_{t-1}, X_{t-1}^{*}}$ from the observed $L_{Y_{t}, m_{t}, \omega \mid m_{t-1}, Y_{t-2}}$ requires

Assumption 4 For a known $\omega(\cdot)$ and any $\left(m_{t}, m_{t-1}\right) \in \operatorname{supp}\left(M_{t}, M_{t-1}\right)$,

(i) $L_{Y_{t}, m_{t}, \omega \mid m_{t-1}, Y_{t-2}}$ is one-to-one;

(ii) $L_{Y_{t} \mid m_{t}, m_{t-1}, X_{t-1}^{*}}$ is one-to-one.

Assumption 4(ii) rules out cases where $Y_{t}$ is discrete-valued, but $X_{t-1}^{*}$ is continuous. Hence, 
in this section, we are restricting attention to games with continuous action spaces, so that $\left(Y_{t}, X_{t-1}^{*}\right)$ are both continuous. ${ }^{1}$

Remark: Instead of proving that the operators are one-to-one in examples 1 and 2, here we only discuss whether the variation in $Y_{t-2}$ or $X_{t-1}^{*}$ will imply variation in $Y_{t}$ for fixed $\left(m_{t}, m_{t-1}\right)$, which is necessary in order for the one-to-one assumptions to hold. In both examples 1 and 2, equation (12) implies that, just holding $m_{t}, y_{t-1}$, and and $m_{t-1}$ fixed, variation in $Y_{t-2}$ will affect $X_{t-1}^{*}$. In turn, changes in $X_{t-1}^{*}$ will induce changes in $X_{t}^{*}$, which will directly affect $Y_{t}$. This reasoning implies that Assumption 4(i) should hold as long as $E\left[\omega\left(Y_{t-1}\right) \mid m_{t}, m_{t-1}, x_{t-1}^{*}\right]$ does not vanish for a nontrivial set of $x_{t-1}^{*}$.

Similarly, variation in $X_{t-1}^{*}$ implies changes in $X_{t}^{*}$, which directly affects $Y_{t}$, even when $m_{t}$ and $m_{t-1}$ are fixed. Therefore, Assumption 4(ii) should hold. However, note that in order for one-to-one to hold, the supports of $Y_{t}$ and $X_{t}^{*}$ should be of the same dimension. If, for example, $Y_{t}$ is discrete (as in entry games), but $X_{t}^{*}$ is continuous, then the condition would fail.

As shown in the Appendix, equation (12) can be written in operator notation as (for fixed $\left.\left(m_{t}, m_{t-1}\right)\right)$ :

$$
L_{Y_{t}, m_{t}, \omega \mid m_{t-1}, Y_{t-2}}=L_{Y_{t} \mid m_{t}, m_{t-1}, X_{t-1}^{*}} D_{\omega \mid m_{t}, m_{t-1}, X_{t-1}^{*}} D_{m_{t} \mid m_{t-1}, X_{t-1}^{*}} L_{X_{t-1}^{*} \mid m_{t-1}, Y_{t-2}}
$$

where $D_{\omega \mid m_{t}, m_{t-1}, X_{t-1}^{*}}$ and $D_{m_{t} \mid m_{t-1}, X_{t-1}^{*}}$ are diagonal operators.

Since $\omega(\cdot)$ is a user-specified function, we can evaluate equation (12) with $\omega(x)=1$, which yields

$$
f_{Y_{t}, M_{t} \mid M_{t-1}, Y_{t-2}}=\int f_{Y_{t} \mid M_{t}, M_{t-1}, X_{t-1}^{*}} f_{M_{t} \mid M_{t-1}, X_{t-1}^{*}} f_{X_{t-1}^{*} \mid M_{t-1}, Y_{t-2}} d x_{t-1}^{*}
$$

The above can be expressed in operator notation as

$$
L_{Y_{t}, m_{t} \mid m_{t-1}, Y_{t-2}}=L_{Y_{t} \mid m_{t}, m_{t-1}, X_{t-1}^{*}} D_{m_{t} \mid m_{t-1}, X_{t-1}^{*}} L_{X_{t-1}^{*}} \mid m_{t-1}, Y_{t-2}
$$

for any given $\left(m_{t}, m_{t-1}\right) \in \operatorname{supp}\left(M_{t}, M_{t-1}\right)$. (See the appendix for the full definition of these operators.)

\footnotetext{
${ }^{1}$ On the other hand, if $X_{t-1}^{*}$ is discrete, one may allow $Y_{t}$ to be discrete with a support no smaller than that of $X_{t-1}^{*}$.
} 
As in $\mathrm{Hu}$ and Schennach (2008), if the latter operator relation can be inverted (which is ensured by Assumption 4), we can combine Eqs. (13) and (15) to get

$$
\begin{aligned}
& L_{Y_{t}, m_{t}, \omega \mid m_{t-1}, Y_{t-2}} L_{Y_{t}, m_{t} \mid m_{t-1}, Y_{t-2}}^{-1} \\
\equiv & L_{Y_{t} \mid m_{t}, m_{t-1}, X_{t-1}^{*}} \cdot D_{\omega \mid m_{t}, m_{t-1}, X_{t-1}^{*}} \cdot L_{Y_{t} \mid m_{t}, m_{t-1}, X_{t-1}^{*}}^{-1} .
\end{aligned}
$$

This representation shows that an eigenvalue-eigenfunction decomposition of the observed operator $L_{Y_{t}, m_{t}, \omega \mid m_{t-1}, Y_{t-2}} L_{Y_{t}, m_{t} \mid m_{t-1}, Y_{t-2}}^{-1}$ yields the unknown density functions $f_{Y_{t} \mid m_{t}, m_{t-1}, X_{t-1}^{*}}$ as the eigenfunctions and $E\left[\omega\left(Y_{t-1}\right) \mid m_{t}, m_{t-1}, x_{t-1}^{*}\right]$ as the eigenvalues.

The following assumption ensures the uniqueness of this decomposition, and restricts the choice of the $\omega(\cdot)$ function.

Assumption 5 (i) For any $\left(m_{t}, m_{t-1}, x_{t-1}^{*}\right) \in \operatorname{supp}\left(M_{t}, M_{t-1}, X_{t-1}^{*}\right)$, there exists a known $\omega(\cdot)$ such that

$$
\left|E\left[\omega\left(Y_{t-1}\right) \mid m_{t}, m_{t-1}, x_{t-1}^{*}\right]\right|<\infty
$$

(ii) For any $\left(m_{t}, m_{t-1}, x_{t-1}^{*}\right)$ and $\left(m_{t}, m_{t-1}, \widetilde{x}_{t-1}^{*}\right)$ in $\operatorname{supp}\left(M_{t}, M_{t-1}, X_{t-1}^{*}\right)$ with $x_{t-1}^{*} \neq$ $\widetilde{x}_{t-1}^{*}$, there exists a known $\omega(\cdot)$ such that

$$
E\left[\omega\left(Y_{t-1}\right) \mid m_{t}, m_{t-1}, x_{t-1}^{*}\right] \neq E\left[\omega\left(Y_{t-1}\right) \mid m_{t}, m_{t-1}, \widetilde{x}_{t-1}^{*}\right]
$$

where both expectations are nonzero.

Assumption 5(i) implies that the conditional expectation $E\left[\omega\left(Y_{t-1}\right) \mid m_{t}, m_{t-1}, x_{t-1}^{*}\right]$ is always bounded. Assumption 5(ii) implies that a change in $x_{t-1}^{*}$ can be detected by the conditional expectation of $\omega\left(Y_{t-1}\right)$ for some known function $\omega(\cdot)$.

Remark: For example 1, as long as the density $f_{Y_{1, t-1} \mid M_{t}, M_{t-1}, X_{t-1}^{*}}$ has a finite mean, we may use $\omega\left(y_{t-1}\right)=y_{1, t-1}$ to satisfy assumption 5(i). When we use $\omega\left(y_{t-1}\right)=I\left(y_{1, t-1}>c\right)$, $E\left[\omega\left(Y_{t-1}\right) \mid m_{t}, m_{t-1}, x_{t-1}^{*}\right]=\operatorname{Pr}\left(Y_{1, t-1}>c \mid m_{t}, m_{t-1}, x_{t-1}^{*}\right)$ also satisfies assumption 5(i).

On the other hand, $M_{t} \mid M_{t-1}, Y_{t-1}$ is deterministic for example 2. Hence, for fixed $\left(m_{t}, m_{t-1}\right)$, the density $f_{Y_{t-1} \mid M_{t}, M_{t-1}, X_{t-1}^{*}}$ is only defined at a single point $y_{t-1}=m_{t}-(1-\delta) m_{t-1}$, which is finite. Therefore, in this example, $E\left[\omega\left(Y_{t-1}\right) \mid m_{t}, m_{t-1}, x_{t-1}^{*}\right]$ is trivially bounded, for $\omega\left(Y_{t-1}\right)=Y_{t-1}$. 
Assumption 5(ii) requires that the conditional expectation $E\left[\omega\left(Y_{t-1}\right) \mid m_{t}, m_{t-1}, x_{t-1}^{*}\right]$ varies in $X_{t-1}^{*}$ given any fixed $\left(m_{t}, m_{t-1}\right)$, so that the "eigenvalues" in the decomposition (16) are distinctive. For example 1, given the preceding discussion, if assumption 5(i) is satisfied, then assumption 5(ii) will hold if, for any $\left(m_{t}, m_{t-1}, x_{t-1}^{*}\right)$ in $\operatorname{supp}\left(M_{t}, M_{t-1}, X_{t-1}^{*}\right)$,

$$
0<\left|E\left[\omega\left(Y_{t-1}\right) \mid m_{t}, m_{t-1}, x_{t-1}^{*}\right]\right|<\infty \text { and } \frac{\partial E\left[\omega\left(Y_{t-1}\right) \mid m_{t}, m_{t-1}, x_{t-1}^{*}\right]}{\partial x_{t-1}^{*}} \neq 0 .
$$

For example 2 , the capital stock $M_{t}$ evolves deterministically, so that $E\left[\omega\left(Y_{t-1}\right) \mid m_{t}, m_{t-1}, x_{t-1}^{*}\right]=$ $\omega\left(m_{t}-(1-\delta) m_{t-1}\right)$. Since this does not change with $x_{t-1}^{*}$ for any fixed $\left(m_{t}, m_{t-1}\right)$, Therefore, assumption $5($ ii) fails.

Remark (Deterministic choices): In some models, the choice variable $Y_{i t}$ is a deterministic function of the current state variables, i.e.,

$$
Y_{i, t-1}=g_{i}\left(M_{t-1}, X_{t-1}^{*}\right), \quad i=1,2 .
$$

In examples 1 and 2, this would be the case if we eliminated the privately-observed demand shocks $\eta_{1 t}$ and $\eta_{2 t}$.

With $\omega\left(y_{t-1}\right)=y_{1, t-1}$, we have

$$
E\left[\omega\left(Y_{t-1}\right) \mid m_{t}, m_{t-1}, x_{t-1}^{*}\right]=g_{1}\left(m_{t-1}, x_{t-1}^{*}\right) .
$$

Therefore, a sufficient condition for assumption 5 is that for any $m_{t-1}$ and $x_{t-1}^{*}$,

$$
0<\left|g_{1}\left(m_{t-1}, x_{t-1}^{*}\right)\right|<\infty \text { and } \frac{\partial g_{1}\left(m_{t-1}, x_{t-1}^{*}\right)}{\partial x_{t-1}^{*}} \neq 0
$$

That means $Y_{t-1}$ is monotonic in $X_{t-1}^{*}$ for any given $m_{t-1}$.

Remark: Notice that in the decomposition (16), the function $\omega(\cdot)$ only appears in the eigenvalues. Therefore, if there are several $\omega(\cdot)$ functions which satisfy Assumption (5), the decompositions (16) using these different $\omega(\cdot)$ 's should yield the same eigenfunctions. Hence, depending on the specific model, it may be possible to use this feature as a general specification check for Assumptions (1) and (2). We do not explore this possibility here.

Under the foregoing assumptions, the density $Y_{t}, m_{t}, y_{t-1} \mid m_{t-1}, Y_{t-2}$ and the function $\omega(\cdot)$ 
(for fixed $m_{t}, m_{t-1}$ ) can form a unique eigenvalue-eigenvector decomposition. In this decomposition, the eigenfunction corresponds to the density $f_{Y_{t} \mid m_{t}, m_{t-1}, X_{t-1}^{*}}\left(\cdot \mid m_{t}, m_{t-1}, x_{t-1}^{*}\right)$ which can be written as

$$
f_{Y_{t} \mid m_{t}, m_{t-1}, X_{t-1}^{*}}\left(\cdot \mid m_{t}, m_{t-1}, x_{t-1}^{*}\right)=f_{Y_{1, t}, Y_{2, t} \mid m_{t}, m_{t-1}, X_{1, t-1}^{*}, X_{2, t-1}^{*}}\left(\cdot, \cdot \mid m_{t}, m_{t-1}, x_{1, t-1}^{*}, x_{2, t-1}^{*}\right) .
$$

The eigenvalue-eigenfunction decomposition only identifies this eigenfunction up to some arbitrary ordering of the $\left(x_{1, t-1}^{*}, x_{2, t-1}^{*}\right)$ argument. Hence, in order to pin down the right ordering of $x_{t-1}^{*}$, an additional ordering assumption is required. In our earlier paper ( $\mathrm{Hu}$ and Shum (2008)), where $x_{t}^{*}$ was scalar-valued, a monotonicity assumption sufficed to pin down the ordering of $x_{t}^{*}$. However, in dynamic games, $x_{t-1}^{*}$ is multivariate, so that monotonicity is no longer well-defined.

Consider the marginal density

$$
f_{Y_{i, t} \mid m_{t}, m_{t-1}, X_{1, t-1}^{*}, X_{2, t-1}^{*}}\left(\cdot \mid m_{t}, m_{t-1}, x_{1, t-1}^{*}, x_{2, t-1}^{*}\right),
$$

which can be computed from Eq. (20) above. We make the following ordering assumption:

Assumption 6 (i) for any given $\left(m_{t}, m_{t-1}\right) \in \operatorname{supp}\left(M_{t}, M_{t-1}\right)$ and $x_{2, t}^{*}$, there exist a known functional $G$ such that $G\left[f_{Y_{1, t} \mid M_{t}, M_{t-1}, X_{t-1}^{*}}\left(\cdot \mid m_{t}, m_{t-1}, x_{1, t-1}^{*}, x_{2, t-1}^{*}\right)\right]$ is monotonic in $x_{1, t-1}^{*}$;

(ii) Without loss of generality, we normalize $x_{1, t-1}^{*}$ as

$$
x_{1, t-1}^{*}=G\left[f_{Y_{1, t} \mid M_{t}, M_{t-1}, X_{t-1}^{*}}\left(\cdot \mid m_{t}, m_{t-1}, x_{1, t-1}^{*}, x_{2, t-1}^{*}\right)\right]
$$

(iii) $x_{2, t-1}^{*}$ also satisfies (i) and (ii), i.e.,

$$
x_{2, t-1}^{*}=G\left[f_{Y_{2, t} \mid M_{t}, M_{t-1}, X_{t-1}^{*}}\left(\cdot \mid m_{t}, m_{t-1}, x_{1, t-1}^{*}, x_{2, t-1}^{*}\right)\right]
$$

for any given $\left(m_{t}, m_{t-1}\right)$ and $x_{1, t-1}^{*}$.

Remark: Consider example 1, and firm 1. A sufficient condition for this assumption is that $f_{Y_{1, t} \mid m_{t}, m_{t-1}, X_{1, t-1}^{*}, X_{2, t-1}^{*}}$ is stochastically increasing (in the sense of first-order stochastic dominance) in $x_{1, t-1}^{*}$, for fixed $x_{2, t-1}^{*}$ and $m_{t}, m_{t-1}$. Note that, in the Markov equilibrium, 
$Y_{1, t}$ is a function of $M_{t}, X_{t}^{*}, \eta_{i, t}$. Hence, the distribution of $Y_{1, t} \mid M_{t}, M_{t-1}, X_{1 t-1}^{*}, X_{2, t-1}^{*}$ can be written as:

$$
Y_{i}^{*}\left(X_{1, t}^{*}, X_{2, t}^{*}, m_{t}, \eta_{i, t}\right) \mid m_{t}, m_{t-1}, X_{1, t-1}^{*}, x_{2, t-1}^{*}
$$

We see from the above that, once $m_{t}, m_{t-1}, x_{2, t-1}^{*}$ are fixed then $X_{1, t-1}^{*}$ affects $Y_{1, t}$ only through the conditional distribution of $X_{1, t}^{*} \mid m_{1, t-1}, X_{1, t-1}^{*}$. Hence, assumption 6(i) is satisfied if (i) $Y_{i, t}$ is increasing in $X_{1, t}^{*}$, for fixed $m_{t}, x_{2, t}^{*}, \eta_{1, t}$; and (ii) the distribution $X_{1, t}^{*} \mid m_{1, t-1}, X_{1, t-1}^{*}$ is stochastically increasing in $X_{1, t-1}^{*} \cdot{ }^{2}$ Both (i) and (ii) should be confirmed on a modelby-model basis, but is not unreasonable given the interpretation of $Y_{i, t}$ as a price and $X_{1, t}^{*}$ as a marginal cost variable.

From the eigenvalue-eigenvector decomposition in Eq. (16), we can identify the densities $f_{Y_{t} \mid m_{t}, m_{t-1}, X_{t-1}^{*}}$. Eq. (11) and assumption 4(ii) then imply the identification of the joint density $f_{m_{t}, y_{t-1}, X_{t-1}^{*} \mid m_{t-1}, Y_{t-2}}$. This can be factored into the components $f_{m_{t}, y_{t-1} \mid m_{t-1}, X_{t-1}^{*}}$, and $f_{X_{t-1}^{*} \mid m_{t-1}, Y_{t-2}}$, for all $\left(y_{t-1}, m_{t}, m_{t-1}\right)$ with positive support. From the factorization

$$
f_{M_{t}, Y_{t-1} \mid M_{t-1}, X_{t-1}^{*}}=f_{M_{t} \mid Y_{t-1}, M_{t-1}, X_{t-1}^{*}} \cdot f_{Y_{t-1} \mid M_{t-1}, X_{t-1}^{*}}
$$

we can recover $f_{M_{t} \mid Y_{t-1}, M_{t-1}, X_{t-1}^{*}}$ and $f_{Y_{t-1} \mid M_{t-1}, X_{t-1}^{*}}$. Given stationarity, the latter density is identical to $f_{Y_{t} \mid M_{t}, X_{t}^{*}}$, so that from $f_{M_{t}, Y_{t-1} \mid M_{t-1}, X_{t-1}^{*}}$ we have recovered the first two components of $f_{W_{t}, X_{t}^{*} \mid W_{t-1}, X_{t-1}^{*}}$ in Eq. (10).

All that remains now is to identify the third component $f_{X_{t}^{*} \mid M_{t}, M_{t-1}, X_{t-1}^{*}}$. To obtain this, note that the following operator relation holds (see the Appendix for details):

$$
f_{Y_{t} \mid m_{t}, m_{t-1}, X_{t-1}^{*}}=L_{Y_{t} \mid m_{t}, X_{t}^{*}} f_{X_{t}^{*} \mid m_{t}, m_{t-1}, X_{t-1}^{*}}
$$

for given $\left(m_{t}, m_{t-1}\right)$, and where we define the operator $L_{Y_{t} \mid m_{t}, X_{t}^{*}}: \mathcal{L}^{p}\left(\mathcal{X}_{t}^{*}\right) \rightarrow \mathcal{L}^{p}\left(\mathcal{Y}_{t}\right)$,

$$
\left(L_{Y_{t} \mid m_{t}, X_{t}^{*}} h\right)(x)=\int f_{Y_{t} \mid M_{t}, X_{t}^{*}}\left(x \mid m_{t}, x_{t}^{*}\right) h\left(x_{t}^{*}\right) d x_{t}^{*}
$$

Identification of $f_{X_{t}^{*} \mid M_{t}, M_{t-1}, X_{t-1}^{*}}$ then requires

Assumption 7 for any $m_{t} \in \mathcal{M}_{t}, L_{Y_{t} \mid m_{t}, X_{t}^{*}}$ is one-to-one.

\footnotetext{
${ }^{2}$ Note that we do not have to worry about an indirect effect of $X_{1 t-1}^{*}$ on $Y_{1 t}$ through the $X_{2 t-1}^{*}$. This is because, for example 1, the evolution of $X_{2 t}^{*}$ depends just on $M_{t}$ and $X_{2 t-1}^{*}$ (cf. Eq. (4)), which are both held fixed for Assumption 5 .
} 
Similarly to assumption 4(ii), this one-to-one assumption rules out the case where the support of $Y_{t}$ is discrete, but $X_{t}^{*}$ is continuous.

Given assumption (7), the final component in Eq. (10) can be recovered as:

$$
f_{X_{t}^{*} \mid m_{1}, m_{t-1}, X_{t-1}^{*}}=\left(L_{Y_{t} \mid m_{t}, X_{t}^{*}}\right)^{-1} f_{Y_{t} \mid m_{t}, m_{t-1}, X_{t-1}^{*}}
$$

where both terms on the right-hand-side have already been identified in previous steps.

Remark (deterministic choices, continued): For the case where $Y_{i t}$ is determinisitc given the current state variables $\left(X_{t}^{*}, M_{t}\right)$, and $y_{i t}=g_{i}\left(m_{t}, x_{t}^{*}\right)$, for both firms $i=1,2$, we can take:

$$
f_{Y_{t} \mid m_{t}, X_{t}^{*}}=\mathbf{1}\left(Y_{i t}=g_{i}\left(m_{t}, X_{t}^{*}\right), i=1,2\right)
$$

Given knowledge of $f_{m_{t}, y_{t-1} \mid m_{t-1}, X_{t-1}^{*}}$, the functions $g_{1}(\cdots)$ and $g_{2}(\cdots)$ can be mapped out by varying $\left(y_{t-1}, m_{t}, m_{t-1}\right)$ along points with positive support. In this case, Assumption 7 requires that the mapping $\left(Y_{1 t}, Y_{2 t}\right)^{\prime}=\left[g_{1}\left(m_{t}, X_{t}^{*}\right), g_{2}\left(m_{t}, X_{t}^{*}\right)\right]^{\prime}$ is invertible in $X_{t}^{*}=$ $\left(X_{1 t}^{*}, X_{2 t}^{*}\right)$. Consequently, equation (21) is analogous to the usual multivariate change of variables formula between $Y_{t}$ and $X_{t}^{*}$.

Finally, we summarize the identification results as follows:

Theorem 1 (Stationary case) Under the assumptions 1, 2, 3, 4, 5, 6, and 7, the density $f_{W_{t}, W_{t-1}, W_{t-2}}$, for any $t \in\{3, \ldots T\}$, uniquely determines the time-invariant Markov equilibrium transition density $f_{W_{2}, X_{2}^{*} \mid W_{1}, X_{1}^{*}}$.

Proof. See the appendix.

This theorem implies that we may identify the Markov kernel density with three periods of data.

Without stationarity, the desired density $f_{Y_{t} \mid M_{t}, X_{t}^{*}}$ is not the same as $f_{Y_{t-1} \mid M_{t-1}, X_{t-1}^{*}}$, which can be recovered from the three observations $f_{W_{t}, W_{t-1}, W_{t-2}}$. However, in this case, we can repeat the whole foregoing argument for the three observations $f_{W_{t+1}, W_{t}, W_{t-1}}$ to identify $f_{Y_{t} \mid M_{t}, X_{t}^{*}}$. Hence, the following corollary is immediate:

Corollary 1 (Nonstationary case) Under the assumptions 1, 2, 4, 5, 6, and 7, the den- 
sity $f_{W_{t+1}, W_{t}, W_{t-1}, W_{t-2}}$ uniquely determines the time-varying Markov equilibrium transition density $f_{W_{t}, X_{t}^{*} \mid W_{t-1}, X_{t-1}^{*}}$, for every period $t \in\{3, \ldots T-1\}$.

\section{Extensions}

\subsection{Games with discrete actions}

Up to this point, we have focused on games where $Y_{t}$ and $X_{t-1}^{*}$ are both continuous, so that assumption 4(ii) is satisfied. In this section, we consider discrete-choice games, where $Y_{t}$ is discrete-valued, but the unobserved state variables $X_{t}^{*}$ are continuous. ${ }^{3}$ In this case, Assumption 4(ii) is violated, so that $\left(Y_{t}, Y_{t-2}\right)$ cannot be used as the two measurements in the $\mathrm{Hu}$ and Schennach (2008) setup.

However, under assumptions 1-2, we may use $\left(M_{t+1}, M_{t-2}\right)$ to play the role of $\left(Y_{t}, Y_{t-2}\right)$ in the identification procedure, especially when $Y_{t}$ is discrete. Consider the joint density of $\left\{M_{t+1}, Y_{t}, M_{t}, Y_{t-1}, M_{t-1}, Y_{t-2}, M_{t-2}\right\}$. One can show that assumptions 1-2 imply that

$$
\begin{aligned}
& f_{M_{t+1}, Y_{t}, M_{t}, Y_{t-1}, M_{t-1}, Y_{t-2}, M_{t-2}} \\
= & \int f_{M_{t+1}, Y_{t} \mid M_{t}, M_{t-1}, X_{t-1}^{*}} f_{M_{t}, Y_{t-1} \mid M_{t-1}, X_{t-1}^{*}} f_{X_{t-1}^{*}, M_{t-1}, Y_{t-2}, M_{t-2}} d x_{t-1}^{*} .
\end{aligned}
$$

The identification strategy in Hu and Schennach (2008) implies that two measurements and a dependent variable of a latent explanatory variable are enough to achieve identification. For fixed $\left(m_{t}, m_{t-1}\right)$, Equation (22) implies that, when $X_{t-1}^{*}, Y_{t}$ and $M_{t}$ are all continuous, we may use either $\left(Y_{t}, Y_{t-2}\right)$ or $\left(M_{t+1}, M_{t-2}\right)$ as the two measurements of $X_{t-1}^{*}$. The former case was considered in the previous section.

However, when $Y_{t}$ is discrete and $M_{t}$ is continuous, we have to use $\left(M_{t+1}, M_{t-2}\right)$ as the two measurements of $X_{t-1}^{*} \cdot{ }^{4}$

\footnotetext{
${ }^{3}$ This is also the setup in Heckman and Navarro's (2007) single-agent dynamic treatment effect model, where $Y_{t}$ is an indicator for school attendance and $X_{t}^{*}$ denotes a student's unobserved ability. In that model, Heckman and Navarro allow $X_{t}^{*}$ to be arbitrarily correlated over time, but assume that it enters the model additively. They demonstrate identification using large support assumptions. In contrast, we restrict the joint process of $W_{t}, X_{t}^{*}$ to be first-order Markov, but allow the unobservables $X_{t}^{*}$ to enter in nonseparable manner. Consequently, our identification arguments are quite different from theirs.

${ }^{4}$ In general, one can use $(U, V)$ as the two measurements of $X_{t-1}^{*}$, where $U=g\left(M_{t+1}, Y_{t}\right)$ and $V=$ $q\left(M_{t-2}, Y_{t-2}\right)$ for some known functions $g(\cdot, \cdot)$ and $q(\cdot, \cdot)$.
} 
Integrating out $\left(Y_{t}, Y_{t-2}\right)$ in Eq. (22), we obtain

$$
\begin{aligned}
& \int \omega\left(y_{t-1}\right) f_{M_{t+1}, M_{t}, Y_{t-1} \mid M_{t-1}, M_{t-2}}\left(x, m_{t}, y_{t-1} \mid m_{t-1}, z\right) d y_{t-1} \\
= & \int\left[f_{M_{t+1} \mid M_{t}, M_{t-1}, X_{t-1}^{*}}\left(x \mid m_{t}, m_{t-1}, x_{t-1}^{*}\right) E\left[\omega\left(Y_{t-1}\right) \mid m_{t}, m_{t-1}, x_{t-1}^{*}\right] .\right. \\
& \left.f_{M_{t}, \mid M_{t-1}, X_{t-1}^{*}}\left(m_{t}, \mid m_{t-1}, x_{t-1}^{*}\right) f_{X_{t-1}^{*} \mid M_{t-1}, M_{t-2}}\left(x_{t-1}^{*} \mid m_{t-1}, z\right)\right] d x_{t-1}^{*}
\end{aligned}
$$

for any $\left(x, m_{t}, m_{t-1}, z\right) \in \operatorname{supp}\left(M_{t+1}, M_{t}, M_{t-1}, M_{t-2}\right)$. We may achieve identification under the assumptions as follows. Assumption 4 is replaced by the following assumption.

Assumption 4' For a known $\omega(\cdot)$ and any $\left(m_{t}, m_{t-1}\right) \in \operatorname{supp}\left(M_{t}, M_{t-1}\right)$,

(i) $L_{M_{t+1}, m_{t}, \omega \mid m_{t-1}, M_{t-2}}$ is one-to-one;

(ii) $L_{M_{t+1} \mid m_{t}, m_{t-1}, X_{t-1}^{*}}$ is one-to-one.

The operators $L_{M_{t+1}, m_{t}, \omega \mid m_{t-1}, M_{t-2}}$ and $L_{M_{t+1} \mid m_{t}, m_{t-1}, X_{t-1}^{*}}$ are defined in the appendix. In the setting where $Y_{t-1}$ is discrete, but $X_{t-1}^{*}$ is continuous, Assumption 4'(ii) essentially requires that the observed state variable $M_{t}$ be informative on $X_{t-1}^{*}$, i.e.,

$$
f_{M_{t} \mid Y_{t-1}, M_{t-1}, X_{t-1}^{*}} \neq f_{M_{t} \mid Y_{t-1}, M_{t-1}} .
$$

This is because, once $\left(m_{t}, m_{t-1}\right)$ are fixed, the effect of $X_{t-1}^{*}$ on $M_{t+1}$ must come either through $Y_{t}$ (which depends on $X_{t}^{*}$, and which in turn is serially correlated with $\left.X_{t-1}^{*}\right)$ ), or through $X_{t}^{*}$ (which is serially correlated with $X_{t-1}^{*}$ ). When $Y_{t}$ is discrete, but $X_{t}^{*}$ is continuous, then $Y_{t}$ cannot fully "transmit" all the information in $X_{t-1}^{*}$, which is required to satisfy Assumption 4'(ii). Hence, $M_{t}$ must transmit the information in $X_{t-1}^{*}$, as in equation (24).

The eigenfunction corresponds to the density $f_{M_{t+1} \mid m_{t}, m_{t-1}, X_{t-1}^{*}}\left(\cdot \mid m_{t}, m_{t-1}, \cdot\right)$ which can be written as

$$
f_{M_{t+1} \mid m_{t}, m_{t-1}, X_{t-1}^{*}}\left(\cdot \mid m_{t}, m_{t-1}, \cdot\right)=f_{M_{1, t+1}, M_{2, t+1} \mid m_{t}, m_{t-1}, X_{1, t-1}^{*}, X_{2, t-1}^{*}}\left(\cdot, \cdot \mid m_{t}, m_{t-1}, \cdot, \cdot\right) .
$$

We then need the following ordering assumption instead of assumption 6 .

Assumption 6' (i) for any given $\left(m_{t}, m_{t-1}\right) \in \operatorname{supp}\left(M_{t}, M_{t-1}\right)$ and $x_{2, t}^{*}$, there exist a 
known functional $G$ such that $G\left[f_{M_{1, t+1} \mid M_{t}, M_{t-1}, X_{t-1}^{*}}\left(\cdot \mid m_{t}, m_{t-1}, x_{1, t-1}^{*}, x_{2, t-1}^{*}\right)\right]$ is monotonic in $x_{1, t-1}^{*}$;

(ii) Without loss of generality, we normalize $x_{1, t-1}^{*}$ as

$$
x_{1, t-1}^{*}=G\left[f_{M_{1, t+1} \mid M_{t}, M_{t-1}, X_{t-1}^{*}}\left(\cdot \mid m_{t}, m_{t-1}, x_{1, t-1}^{*}, x_{2, t-1}^{*}\right)\right]
$$

(iii) $x_{2, t-1}^{*}$ also satisfies (i) and (ii), i.e.,

$$
x_{2, t-1}^{*}=G\left[f_{M_{2, t+1} \mid M_{t}, M_{t-1}, X_{t-1}^{*}}\left(\cdot \mid m_{t}, m_{t-1}, x_{1, t-1}^{*}, x_{2, t-1}^{*}\right)\right]
$$

for any given $\left(m_{t}, m_{t-1}\right)$ and $x_{1, t-1}^{*}$.

The following assumption is needed to replace assumption 7 .

Assumption 7' for any $m_{t} \in \mathcal{M}_{t}, L_{M_{t+1} \mid m_{t}, X_{t}^{*}}$ is one-to-one.

The operator $L_{M_{t+1} \mid m_{t}, X_{t}^{*}}$ is defined in the appendix. This assumption also requires that equation (24) holds. Finally, we summarize the identification results as follows:

Theorem 2 Under the assumptions 1, 2, 3, 4', 5, 6', and 7', the density $f_{W_{t+1}, W_{t}, W_{t-1}, W_{t-2}}$ for any $t \in\{3, \ldots T-1\}$, uniquely determines the time-invariant Markov equilibrium tran-

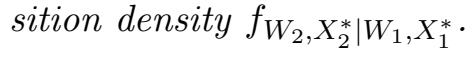

Proof. See the appendix.

\subsection{Alternatives to Assumption 2(ii)}

In this section, we consider alternative conditions of assumption 2(ii). Assumption 2(ii) implies that $X_{t}^{*}$ is independent of $Y_{t-1}$ conditional on $M_{t}, M_{t-1}$ and $X_{t-1}^{*}$. There are other alternative "limited feedback" assumptions, which may be suitable for different empirical 
settings. Assumptions 1 and 2(i) imply

$$
\begin{aligned}
& f_{W_{t+1}, W_{t}, W_{t-1}, W_{t-2}} \\
= & f_{Y_{t+1}, M_{t+1}, Y_{t}, M_{t}, Y_{t-1}, M_{t-1}, Y_{t-2}, M_{t-2}} \\
= & \iint\left[f_{Y_{t+1}, M_{t+1} \mid Y_{t}, M_{t}, X_{t}^{*}} f_{Y_{t} \mid M_{t}, X_{t}^{*}} f_{X_{t}^{*}, M_{t} \mid Y_{t-1}, M_{t-1}, X_{t-1}^{*}}\right. \\
& \left.f_{Y_{t-1} \mid M_{t-1}, X_{t-1}^{*}} f_{X_{t-1}^{*}, M_{t-1}, Y_{t-2}, M_{t-2}}\right] d x_{t}^{*} d x_{t-1}^{*} .
\end{aligned}
$$

Assumption 2(ii) implies that the state transition density satisfies

$$
f_{X_{t}^{*}, M_{t} \mid Y_{t-1}, M_{t-1}, X_{t-1}^{*}}=f_{X_{t}^{*} \mid M_{t}, M_{t-1}, X_{t-1}^{*}} f_{M_{t} \mid Y_{t-1}, M_{t-1}, X_{t-1}^{*}}
$$

Alternative "limited feedback" assumptions may be imposed on the density $f_{X_{t}^{*}, M_{t} \mid Y_{t-1}, M_{t-1}, X_{t-1}^{*}}$. One alternative to assumption 2(ii) is

$$
f_{X_{t}^{*}, M_{t} \mid Y_{t-1}, M_{t-1}, X_{t-1}^{*}}=f_{X_{t}^{*} \mid M_{t}, Y_{t-1}, X_{t-1}^{*}} f_{M_{t} \mid Y_{t-1}, M_{t-1}, X_{t-1}^{*}}
$$

which implies that $M_{t-1}$ does not have a direct effect on $X_{t}^{*}$ conditional on $M_{t}, Y_{t-1}$, and $X_{t-1}^{*}$. A second alternative is

$$
f_{X_{t}^{*}, M_{t} \mid Y_{t-1}, M_{t-1}, X_{t-1}^{*}}=f_{M_{t} \mid X_{t}^{*}, Y_{t-1}, M_{t-1}} f_{X_{t}^{*} \mid Y_{t-1}, M_{t-1}, X_{t-1}^{*}}
$$

which is the "limited feedback" assumption used in our earlier study (Hu and Shum (2008)) of identification on single-agent dynamic optimization problems. Both alternatives (25) and (26) can be handled using identification arguments similar to the one in $\mathrm{Hu}$ and Shum (2008).

A third alternative to assumption 2(ii) is

$$
f_{X_{t}^{*}, M_{t} \mid Y_{t-1}, M_{t-1}, X_{t-1}^{*}}=f_{X_{t}^{*} \mid M_{t}, Y_{t-1}, M_{t-1}, X_{t-1}^{*}} f_{M_{t} \mid M_{t-1}, X_{t-1}^{*}} .
$$

This alternative can be handled in an identification framework similar to the one used in this paper. 


\section{Conclusions}

In this paper, we show several results regarding nonparametric identification in a general class of Markov dynamic games, including many models in the Ericson and Pakes (1995) and Pakes and McGuire (1994) framework. We show that only three observations $W_{t}, \ldots, W_{t-2}$ are required to identify $W_{t}, X_{t}^{*} \mid W_{t-1}, X_{t-1}^{*}$ in the stationary case, when $Y_{t}$ is a continuous choice variable. If $Y_{t}$ is a discrete choice variable (while $X_{t}^{*}$ is continuous), then four observations are required for identification.

In ongoing work, we are working on developing estimation procedures for dynamic games which utilize these identification results. 
Proof. (theorem 1) First, assumptions 1-2 imply that the density of interest becomes

$$
\begin{aligned}
f_{W_{t}, X_{t}^{*} \mid W_{t-1}, X_{t-1}^{*}} & =f_{Y_{t}, M_{t}, X_{t}^{*} \mid Y_{t-1}, M_{t-1}, X_{t-1}^{*}} \\
& =f_{Y_{t} \mid M_{t}, X_{t}^{*}, Y_{t-1}, M_{t-1}, X_{t-1}^{*}} f_{X_{t}^{*} \mid M_{t}, Y_{t-1}, M_{t-1}, X_{t-1}^{*}} f_{M_{t} \mid Y_{t-1}, M_{t-1}, X_{t-1}^{*}} \\
& =f_{Y_{t} \mid M_{t}, X_{t}^{*}} f_{X_{t}^{*} \mid M_{t}, M_{t-1}, X_{t-1}^{*}} f_{M_{t} \mid Y_{t-1}, M_{t-1}, X_{t-1}^{*}} .
\end{aligned}
$$

We consider the observed density $f_{W_{t+1}, W_{t}, W_{t-1}, W_{t-2}}$. One can show that assumptions 1 and 2(i) imply

$$
\begin{aligned}
& f_{W_{t+1}, W_{t}, W_{t-1}, W_{t-2}} \\
= & \iint f_{W_{t+1}, W_{t}, W_{t-1}, W_{t-2}, X_{t}^{*}, X_{t-1}^{*}} d x_{t}^{*} d x_{t-1}^{*} \\
= & \iint f_{W_{t+1} \mid W_{t}, W_{t-1}, W_{t-2}, X_{t}^{*}, X_{t-1}^{*}} f_{W_{t}, X_{t}^{*} \mid W_{t-1}, W_{t-2}, X_{t-1}^{*}} f_{W_{t-1}, W_{t-2}, X_{t-1}^{*}} d x_{t}^{*} d x_{t-1}^{*} \\
= & \iint f_{W_{t+1} \mid W_{t}, X_{t}^{*}} f_{W_{t}, X_{t}^{*} \mid W_{t-1}, X_{t-1}^{*}} f_{W_{t-1}, W_{t-2}, X_{t-1}^{*}} d x_{t}^{*} d x_{t-1}^{*} \\
= & \iint f_{Y_{t+1}, M_{t+1} \mid Y_{t}, M_{t}, X_{t}^{*}} f_{Y_{t} \mid M_{t}, X_{t}^{*}} f_{X_{t}^{*} \mid M_{t}, Y_{t-1}, M_{t-1}, X_{t-1}^{*}} f_{M_{t} \mid Y_{t-1}, M_{t-1}, X_{t-1}^{*}} f_{X_{t-1}^{*}, Y_{t-1}, M_{t-1}, Y_{t-2}, M_{t-2}} d x_{t}^{*} d x_{t-1}^{*} \\
= & \iint f_{Y_{t+1}, M_{t+1} \mid Y_{t}, M_{t}, X_{t}^{*}} f_{Y_{t} \mid M_{t}, X_{t}^{*}} f_{X_{t}^{*} \mid M_{t}, Y_{t-1}, M_{t-1}, X_{t-1}^{*}} f_{M_{t} \mid Y_{t-1}, M_{t-1}, X_{t-1}^{*}} \times \\
& \times f_{Y_{t-1} \mid M_{t-1}, X_{t-1}^{*}} f_{X_{t-1}^{*}, M_{t-1}, Y_{t-2}, M_{t-2}} d x_{t}^{*} d x_{t-1}^{*} \\
= & \iint f_{Y_{t+1}, M_{t+1} \mid Y_{t}, M_{t}, X_{t}^{*}} f_{Y_{t} \mid M_{t}, X_{t}^{*}} f_{X_{t}^{*} \mid M_{t}, Y_{t-1}, M_{t-1}, X_{t-1}^{*}} f_{M_{t}, Y_{t-1} \mid M_{t-1}, X_{t-1}^{*}} f_{X_{t-1}^{*}, M_{t-1}, Y_{t-2}, M_{t-2}} d x_{t}^{*} d x_{t-1}^{*} .
\end{aligned}
$$

Assumption 2(ii) then implies

$$
\begin{aligned}
& f_{W_{t+1}, W_{t}, W_{t-1}, W_{t-2}} \\
= & \iint f_{Y_{t+1}, M_{t+1} \mid Y_{t}, M_{t}, X_{t}^{*}} f_{Y_{t} \mid M_{t}, X_{t}^{*}} f_{X_{t}^{*} \mid M_{t}, M_{t-1}, X_{t-1}^{*}} f_{M_{t}, Y_{t-1} \mid M_{t-1}, X_{t-1}^{*}} f_{X_{t-1}^{*}, M_{t-1}, Y_{t-2}, M_{t-2}} d x_{t}^{*} d x_{t-1}^{*} \\
= & \int\left(\int f_{Y_{t+1}, M_{t+1} \mid Y_{t}, M_{t}, X_{t}^{*}} f_{Y_{t} \mid M_{t}, X_{t}^{*}} f_{X_{t}^{*} \mid M_{t}, M_{t-1}, X_{t-1}^{*}} d x_{t}^{*}\right) f_{M_{t}, Y_{t-1} \mid M_{t-1}, X_{t-1}^{*}} f_{X_{t-1}^{*}, M_{t-1}, Y_{t-2}, M_{t-2}} d x_{t-1}^{*}
\end{aligned}
$$


The expression in the parenthesis can be simplified as follows:

$$
\begin{aligned}
& \int f_{Y_{t+1}, M_{t+1} \mid Y_{t}, M_{t}, X_{t}^{*}} f_{Y_{t} \mid M_{t}, X_{t}^{*}} f_{X_{t}^{*} \mid M_{t}, M_{t-1}, X_{t-1}^{*}} d x_{t}^{*} \\
= & \int f_{Y_{t+1}, M_{t+1} \mid Y_{t}, M_{t}, M_{t-1}, X_{t}^{*}, X_{t-1}^{*}} f_{Y_{t} \mid M_{t}, M_{t-1}, X_{t}^{*}, X_{t-1}^{*}} f_{X_{t}^{*} \mid M_{t}, M_{t-1}, X_{t-1}^{*}} d x_{t}^{*} \\
= & \int f_{Y_{t+1}, M_{t+1}, Y_{t}, X_{t}^{*} \mid M_{t}, M_{t-1}, X_{t-1}^{*}} d x_{t}^{*} \\
= & f_{Y_{t+1}, M_{t+1}, Y_{t} \mid M_{t}, M_{t-1}, X_{t-1}^{*}} .
\end{aligned}
$$

We then have

$$
\begin{aligned}
& f_{W_{t+1}, W_{t}, W_{t-1}, W_{t-2}} \\
= & f_{Y_{t+1}, M_{t+1}, Y_{t}, M_{t}, Y_{t-1}, M_{t-1}, Y_{t-2}, M_{t-2}} \\
= & \int f_{Y_{t+1}, M_{t+1}, Y_{t} \mid M_{t}, M_{t-1}, X_{t-1}^{*}} f_{M_{t}, Y_{t-1} \mid M_{t-1}, X_{t-1}^{*}} f_{X_{t-1}^{*}, M_{t-1}, Y_{t-2}, M_{t-2}} d x_{t-1}^{*}
\end{aligned}
$$

Integrating out $Y_{t+1}, M_{t+1}$, and $M_{t-2}$ leads to

$$
\begin{aligned}
& f_{Y_{t}, M_{t}, Y_{t-1} \mid M_{t-1}, Y_{t-2}} \\
= & \int f_{Y_{t} \mid M_{t}, M_{t-1}, X_{t-1}^{*}} f_{M_{t}, Y_{t-1} \mid M_{t-1}, X_{t-1}^{*}} f_{X_{t-1}^{*} \mid M_{t-1}, Y_{t-2}} d x_{t-1}^{*} .
\end{aligned}
$$

Furthermore, we may have for a user-specified function $\omega(\cdot)$

$$
\begin{aligned}
& \int \omega\left(y_{t-1}\right) f_{Y_{t}, M_{t}, Y_{t-1} \mid M_{t-1}, Y_{t-2}} d y_{t-1} \\
= & \int f_{Y_{t} \mid M_{t}, M_{t-1}, X_{t-1}^{*}} E\left[\omega\left(Y_{t-1}\right) \mid m_{t}, m_{t-1}, x_{t-1}^{*}\right] f_{M_{t}, \mid M_{t-1}, X_{t-1}^{*}} f_{X_{t-1}^{*} \mid M_{t-1}, Y_{t-2}} d x_{t-1}^{*},
\end{aligned}
$$

One can show that the equation above is equivalent to

$$
L_{Y_{t}, m_{t}, \omega \mid m_{t-1}, Y_{t-2}}=L_{Y_{t} \mid m_{t}, m_{t-1}, X_{t-1}^{*}} D_{\omega \mid m_{t}, m_{t-1}, X_{t-1}^{*}} D_{m_{t} \mid m_{t-1}, X_{t-1}^{*}} L_{X_{t-1}^{*} \mid m_{t-1}, Y_{t-2}}
$$

where for any given $\left(m_{t}, m_{t-1}\right) \in \operatorname{supp}\left(M_{t}, M_{t-1}\right)$

$$
\begin{aligned}
L_{Y_{t}, m_{t}, \omega \mid m_{t-1}, Y_{t-2}} & : \mathcal{L}^{p}\left(\mathcal{Y}_{t-2}\right) \rightarrow \mathcal{L}^{p}\left(\mathcal{Y}_{t}\right), \\
\left(L_{Y_{t}, m_{t}, \omega \mid m_{t-1}, Y_{t-2}} h\right)(x) & =\int k\left(x, m_{t} \mid m_{t-1}, z\right) h(z) d z, \\
\text { with } k\left(x, m_{t} \mid m_{t-1}, z\right) & =\int \omega\left(y_{t-1}\right) f_{Y_{t}, M_{t}, Y_{t-1} \mid M_{t-1}, Y_{t-2}}\left(x, m_{t}, y_{t-1} \mid m_{t-1}, z\right) d y_{t-1},
\end{aligned}
$$




$$
\begin{aligned}
& L_{Y_{t} \mid m_{t}, m_{t-1}, X_{t-1}^{*}} \quad: \quad \mathcal{L}^{p}\left(\mathcal{X}_{t-1}^{*}\right) \rightarrow \mathcal{L}^{p}\left(\mathcal{Y}_{t}\right), \\
& \left(L_{Y_{t} \mid m_{t}, m_{t-1}, X_{t-1}^{*}} h\right)(x)=\int f_{Y_{t} \mid M_{t}, M_{t-1}, X_{t-1}^{*}}\left(x \mid m_{t}, m_{t-1}, x_{t-1}^{*}\right) h\left(x_{t-1}^{*}\right) d x_{t-1}^{*} \text {, } \\
& D_{\omega \mid m_{t}, m_{t-1}, X_{t-1}^{*}}: \quad \mathcal{L}^{p}\left(\mathcal{X}_{t-1}^{*}\right) \rightarrow \mathcal{L}^{p}\left(\mathcal{X}_{t-1}^{*}\right), \\
& \left(D_{\omega \mid m_{t}, m_{t-1}, X_{t-1}^{*}} h\right)\left(x_{t-1}^{*}\right)=E\left[\omega\left(Y_{t-1}\right) \mid m_{t}, m_{t-1}, x_{t-1}^{*}\right] h\left(x_{t-1}^{*}\right) \text {, } \\
& D_{m_{t} \mid m_{t-1}, X_{t-1}^{*}}: \quad \mathcal{L}^{p}\left(\mathcal{X}_{t-1}^{*}\right) \rightarrow \mathcal{L}^{p}\left(\mathcal{X}_{t-1}^{*}\right), \\
& \left(D_{m_{t} \mid m_{t-1}, X_{t-1}^{*}} h\right)\left(x_{t-1}^{*}\right)=f_{M_{t} \mid M_{t-1}, X_{t-1}^{*}}\left(m_{t} \mid m_{t-1}, x_{t-1}^{*}\right) h\left(x_{t-1}^{*}\right) \text {, } \\
& L_{X_{t-1}^{*} \mid m_{t-1}, Y_{t-2}} \quad: \quad \mathcal{L}^{p}\left(\mathcal{Y}_{t-2}\right) \rightarrow \mathcal{L}^{p}\left(\mathcal{X}_{t-1}^{*}\right), \\
& \left(L_{X_{t-1}^{*} \mid m_{t-1}, Y_{t-2}} h\right)\left(x_{t-1}^{*}\right)=\int f_{X_{t-1}^{*} \mid M_{t-1}, Y_{t-2}}\left(x_{t-1}^{*} \mid m_{t-1}, z\right) h(z) d z .
\end{aligned}
$$

Integrating out $Y_{t-1}$ in equation (30) leads to

$$
\begin{aligned}
& f_{Y_{t}, M_{t} \mid M_{t-1}, Y_{t-2}} \\
= & \int f_{Y_{t} \mid M_{t}, M_{t-1}, X_{t-1}^{*}} f_{M_{t} \mid M_{t-1}, X_{t-1}^{*}} f_{X_{t-1}^{*} \mid M_{t-1}, Y_{t-2}} d x_{t-1}^{*} .
\end{aligned}
$$

This equation is equivalent to

$$
L_{Y_{t}, m_{t} \mid m_{t-1}, Y_{t-2}}=L_{Y_{t} \mid m_{t}, m_{t-1}, X_{t-1}^{*}} D_{m_{t} \mid m_{t-1}, X_{t-1}^{*}} L_{X_{t-1}^{*} \mid m_{t-1}, Y_{t-2}}
$$

for any given $\left(m_{t}, m_{t-1}\right) \in \operatorname{supp}\left(M_{t}, M_{t-1}\right)$, where

$$
\begin{aligned}
L_{Y_{t}, m_{t} \mid m_{t-1}, Y_{t-2}} & : \quad \mathcal{L}^{p}\left(\mathcal{Y}_{t-2}\right) \rightarrow \mathcal{L}^{p}\left(\mathcal{Y}_{t}\right) \\
\left(L_{Y_{t}, m_{t} \mid m_{t-1}, Y_{t-2}} h\right)(x) & =\int f_{Y_{t}, M_{t} \mid M_{t-1}, Y_{t-2}}\left(x, m_{t} \mid m_{t-1}, z\right) h(z) d z
\end{aligned}
$$

Assumption 4 then implies

$$
\begin{aligned}
& L_{Y_{t}, m_{t}, y_{t-1} \mid m_{t-1}, Y_{t-2}} L_{Y_{t}, m_{t} \mid m_{t-1}, Y_{t-2}}^{-1} \\
\equiv & L_{Y_{t} \mid m_{t}, m_{t-1}, X_{t-1}^{*}} D_{\omega \mid m_{t}, m_{t-1}, X_{t-1}^{*}} L_{Y_{t} \mid m_{t}, m_{t-1}, X_{t-1}^{*}}^{*} .
\end{aligned}
$$


With assumptions 3, 4, 5, 6, and 7, theorem 1 in $\mathrm{Hu}$ and Schennach (2008) implies that $f_{Y_{t} \mid M_{t}, M_{t-1}, X_{t-1}^{*}}$ is nonparametrically identified. By equation (30) and assumption 4, identification of $L_{Y_{t} \mid m_{t}, m_{t-1}, X_{t-1}^{*}}$ implies that of $f_{M_{t}, Y_{t-1} \mid M_{t-1}, X_{t-1}^{*}}$ and $f_{X_{t-1}^{*} \mid M_{t-1}, Y_{t-2}}$. We may then identify $f_{Y_{t-1} \mid M_{t-1}, X_{t-1}^{*}}$ and $f_{M_{t} \mid Y_{t-1}, M_{t-1}, X_{t-1}^{*}}$ from

$$
\begin{aligned}
f_{Y_{t-1} \mid M_{t-1}, X_{t-1}^{*}} & =\int f_{M_{t}, Y_{t-1} \mid M_{t-1}, X_{t-1}^{*}} d m_{t}, \\
f_{M_{t} \mid Y_{t-1}, M_{t-1}, X_{t-1}^{*}} & =\frac{f_{M_{t}, Y_{t-1} \mid M_{t-1}, X_{t-1}^{*}}}{f_{Y_{t-1} \mid M_{t-1}, X_{t-1}^{*}}} .
\end{aligned}
$$

By the stationarity assumption 3, we identify $f_{Y_{t} \mid M_{t}, X_{t}^{*}}=f_{Y_{t-1} \mid M_{t-1}, X_{t-1}^{*}}$. In order to identify $f_{X_{t}^{*} \mid M_{t}, M_{t-1}, X_{t-1}^{*}}$, we consider

$$
\begin{aligned}
f_{Y_{t} \mid M_{t}, M_{t-1}, X_{t-1}^{*}} & =\int f_{Y_{t}, X_{t}^{*} \mid M_{t}, M_{t-1}, X_{t-1}^{*}} d x_{t}^{*} \\
& =\int f_{Y_{t} \mid M_{t}, X_{t}^{*}} f_{X_{t}^{*} \mid M_{t}, M_{t-1}, X_{t-1}^{*}} d x_{t}^{*}
\end{aligned}
$$

Notice that $f_{Y_{t} \mid M_{t}, M_{t-1}, X_{t-1}^{*}}$ on the left hand side has been identified. We define for a given $m_{t}$

$$
\begin{aligned}
L_{Y_{t} \mid m_{t}, X_{t}^{*}} & : \mathcal{L}^{p}\left(\mathcal{X}_{t}^{*}\right) \rightarrow \mathcal{L}^{p}\left(\mathcal{Y}_{t}\right) \\
\left(L_{Y_{t} \mid m_{t}, X_{t}^{*}} h\right)(x) & =\int f_{Y_{t} \mid M_{t}, X_{t}^{*}}\left(x \mid m_{t}, x_{t}^{*}\right) h\left(x_{t}^{*}\right) d x_{t}^{*}
\end{aligned}
$$

We then have

$$
f_{Y_{t} \mid M_{t}=m_{t}, M_{t-1}=m_{t-1}, X_{t-1}^{*}}=L_{Y_{t} \mid m_{t}, X_{t}^{*}} f_{X_{t}^{*} \mid M_{t}=m_{t}, M_{t-1}=m_{t-1}, X_{t-1}^{*}} .
$$

Then the invertibility of $L_{Y_{t} \mid m_{t}, X_{t}^{*}}$ in assumption 7 implies the identification of $f_{X_{t}^{*} \mid M_{t}, M_{t-1}, X_{t-1}^{*}}$. In summary, we have identified $f_{Y_{t} \mid M_{t}, X_{t}^{*}}, f_{M_{t} \mid Y_{t-1}, M_{t-1}, X_{t-1}^{*}}$, and $f_{X_{t}^{*} \mid M_{t}, M_{t-1}, X_{t-1}^{*}}$. Therefore, the density of interest $f_{Y_{t}, M_{t}, X_{t}^{*} \mid Y_{t-1}, M_{t-1}, X_{t-1}^{*}}$, i.e., $f_{W_{t}, X_{t}^{*} \mid W_{t-1}, X_{t-1}^{*}}$ is identified in equation (28).

Proof. (theorem 2) Integrating out $Y_{t+1}, Y_{t}$, and $Y_{t-2}$ in equation (29) leads to

$$
\begin{aligned}
& f_{M_{t+1}, M_{t}, Y_{t-1} \mid M_{t-1}, M_{t-2}} \\
= & \int f_{M_{t+1} \mid M_{t}, M_{t-1}, X_{t-1}^{*}} f_{M_{t}, Y_{t-1} \mid M_{t-1}, X_{t-1}^{*}} f_{X_{t-1}^{*}, M_{t-1}, M_{t-2}} d x_{t-1}^{*} .
\end{aligned}
$$


We may then have

$$
\begin{aligned}
& \int \omega\left(y_{t-1}\right) f_{M_{t+1}, M_{t}, Y_{t-1} \mid M_{t-1}, M_{t-2}} d y_{t-1} \\
= & \int f_{M_{t+1} \mid M_{t}, M_{t-1}, X_{t-1}^{*}} E\left[\omega\left(Y_{t-1}\right) \mid m_{t}, m_{t-1}, x_{t-1}^{*}\right] f_{M_{t}, \mid M_{t-1}, X_{t-1}^{*}} f_{X_{t-1}^{*} \mid M_{t-1}, M_{t-2}} d x_{t-1}^{*} .
\end{aligned}
$$

The equation above is equivalent to

$$
L_{M_{t+1}, m_{t}, \omega \mid m_{t-1}, M_{t-2}}=L_{M_{t+1} \mid m_{t}, m_{t-1}, X_{t-1}^{*}} D_{\omega \mid m_{t}, m_{t-1}, X_{t-1}^{*}} D_{m_{t} \mid m_{t-1}, X_{t-1}^{*}} L_{X_{t-1}^{*} \mid m_{t-1}, M_{t-2}},
$$

where for any given $\left(m_{t}, m_{t-1}\right) \in \operatorname{supp}\left(M_{t}, M_{t-1}\right)$

$$
\begin{aligned}
& \left.L_{M_{t+1}, m_{t}, \omega \mid m_{t-1}, M_{t-2}} \quad: \quad \mathcal{L}^{p}\left(\mathcal{M}_{t-2}\right) \rightarrow \mathcal{L}^{p}\left(\mathcal{M}_{t+1}\right)\right), \\
& \left(L_{M_{t+1}, m_{t}, \omega \mid m_{t-1}, M_{t-2}} h\right)(x)=\int k\left(x, m_{t} \mid m_{t-1}, z\right) h(z) d z \\
& k\left(x, m_{t} \mid m_{t-1}, z\right)=\int \omega\left(y_{t-1}\right) f_{M_{t+1}, M_{t}, Y_{t-1} \mid M_{t-1}, M_{t-2}}\left(x, m_{t}, y_{t-1} \mid m_{t-1}, z\right) d y_{t-1} . \\
& L_{M_{t+1} \mid m_{t}, m_{t-1}, X_{t-1}^{*}}: \quad \mathcal{L}^{p}\left(\mathcal{X}_{t-1}^{*}\right) \rightarrow \mathcal{L}^{p}\left(\mathcal{M}_{t+1}\right), \\
& \left(L_{M_{t+1} \mid m_{t}, m_{t-1}, X_{t-1}^{*}} h\right)(x)=\int f_{M_{t+1} \mid M_{t}, M_{t-1}, X_{t-1}^{*}}\left(x \mid m_{t}, m_{t-1}, x_{t-1}^{*}\right) h\left(x_{t-1}^{*}\right) d x_{t-1}^{*} \text {, } \\
& L_{X_{t-1}^{*} \mid m_{t-1}, M_{t-2}} \quad: \quad \mathcal{L}^{p}\left(\mathcal{M}_{t-2}\right) \rightarrow \mathcal{L}^{p}\left(\mathcal{X}_{t-1}^{*}\right) \\
& \left(L_{X_{t-1}^{*} \mid m_{t-1}, M_{t-2}} h\right)\left(x_{t-1}^{*}\right)=\int f_{X_{t-1}^{*} \mid M_{t-1}, M_{t-2}}\left(x_{t-1}^{*} \mid m_{t-1}, z\right) h(z) d z .
\end{aligned}
$$

Letting $\omega(\cdot)=1$ in equation $(35)$ leads to

$$
\begin{aligned}
& f_{M_{t+1}, M_{t} \mid M_{t-1}, M_{t-2}} \\
= & \int f_{M_{t+1} \mid M_{t}, M_{t-1}, X_{t-1}^{*}} f_{M_{t} \mid M_{t-1}, X_{t-1}^{*}} f_{X_{t-1}^{*}, M_{t-1}, M_{t-2}} d x_{t-1}^{*} .
\end{aligned}
$$

This equation is equivalent to

$$
L_{M_{t+1}, m_{t} \mid m_{t-1}, M_{t-2}}=L_{M_{t+1} \mid m_{t}, m_{t-1}, X_{t-1}^{*}} D_{m_{t} \mid m_{t-1}, X_{t-1}^{*}} L_{X_{t-1}^{*} \mid m_{t-1}, M_{t-2}}
$$


for any given $\left(m_{t}, m_{t-1}\right) \in \operatorname{supp}\left(M_{t}, M_{t-1}\right)$, where

$$
\begin{aligned}
L_{M_{t+1}, m_{t} \mid m_{t-1}, M_{t-2}} & \left.: \quad \mathcal{L}^{p}\left(\mathcal{M}_{t-2}\right) \rightarrow \mathcal{L}^{p}\left(\mathcal{M}_{t+1}\right)\right) \\
\left(L_{M_{t+1}, m_{t} \mid m_{t-1}, M_{t-2}} h\right)(x) & =\int f_{M_{t+1}, M_{t} \mid M_{t-1}, M_{t-2}}\left(x, m_{t} \mid m_{t-1}, z\right) h(z) d z .
\end{aligned}
$$

The same identification procedure as in the proof of theorem 1 implies the identification of $f_{M_{t+1} \mid M_{t}, M_{t-1}, X_{t-1}^{*}}, f_{Y_{t-1} \mid M_{t-1}, X_{t-1}^{*}}$ and $f_{M_{t} \mid Y_{t-1}, M_{t-1}, X_{t-1}^{*}}$. By the stationarity, we identify $f_{M_{t+1} \mid Y_{t}, M_{t}, X_{t}^{*}}=f_{M_{t} \mid Y_{t-1}, M_{t-1}, X_{t-1}^{*}}$ and $f_{Y_{t} \mid M_{t}, X_{t}^{*}}=f_{Y_{t-1} \mid M_{t-1}, X_{t-1}^{*}}$, which implies identification of $f_{M_{t+1} \mid M_{t}, X_{t}^{*}}$ through

$$
f_{M_{t+1} \mid M_{t}, X_{t}^{*}}=\int_{Y_{t}} f_{M_{t+1} \mid Y_{t}, M_{t}, X_{t}^{*}} f_{Y_{t} \mid M_{t}, X_{t}^{*}}
$$

In order to identify $f_{X_{t}^{*} \mid M_{t}, M_{t-1}, X_{t-1}^{*}}$, we consider

$$
\begin{aligned}
f_{M_{t+1} \mid M_{t}, M_{t-1}, X_{t-1}^{*}} & =\int_{Y_{t}} \int f_{M_{t+1}, Y_{t}, X_{t}^{*} \mid M_{t}, M_{t-1}, X_{t-1}^{*}} d x_{t}^{*} \\
& =\int_{Y_{t}} \int f_{M_{t+1} \mid Y_{t}, M_{t}, X_{t}^{*}} f_{Y_{t} \mid M_{t}, X_{t}^{*}} f_{X_{t}^{*} \mid M_{t}, M_{t-1}, X_{t-1}^{*}} d x_{t}^{*} \\
& =\int\left(\int_{Y_{t}} f_{M_{t+1} \mid Y_{t}, M_{t}, X_{t}^{*}} f_{Y_{t} \mid M_{t}, X_{t}^{*}}\right) f_{X_{t}^{*} \mid M_{t}, M_{t-1}, X_{t-1}^{*}} d x_{t}^{*} \\
& =\int f_{M_{t+1} \mid M_{t}, X_{t}^{*}} f_{X_{t}^{*} \mid M_{t}, M_{t-1}, X_{t-1}^{*}} d x_{t}^{*} .
\end{aligned}
$$

Notice that $f_{M_{t+1} \mid M_{t}, M_{t-1}, X_{t-1}^{*}}$ on the left hand side has been identified. We define for a given $m_{t}$

$$
\begin{aligned}
L_{M_{t+1} \mid m_{t}, X_{t}^{*}} & : \mathcal{L}^{p}\left(\mathcal{X}_{t}^{*}\right) \rightarrow \mathcal{L}^{p}\left(\mathcal{M}_{t+1}\right) \\
\left(L_{M_{t+1} \mid m_{t}, X_{t}^{*}} h\right)(x) & =\int f_{M_{t+1} \mid M_{t}, X_{t}^{*}}\left(x \mid m_{t}, x_{t}^{*}\right) h\left(x_{t}^{*}\right) d x_{t}^{*}
\end{aligned}
$$

We then have

$$
f_{M_{t+1} \mid M_{t}=m_{t}, M_{t-1}=m_{t-1}, X_{t-1}^{*}}=L_{M_{t+1} \mid m_{t}, X_{t}^{*}} f_{X_{t}^{*} \mid M_{t}=m_{t}, M_{t-1}=m_{t-1}, X_{t-1}^{*}} .
$$

Then the invertibility of $L_{M_{t+1} \mid m_{t}, X_{t}^{*}}$ in assumption $7^{\prime}$ implies the identification of $f_{X_{t}^{*} \mid M_{t}, M_{t-1}, X_{t-1}^{*}}$. In summary, we have identified $f_{Y_{t} \mid M_{t}, X_{t}^{*}}, f_{M_{t} \mid Y_{t-1}, M_{t-1}, X_{t-1}^{*}}$, and $f_{X_{t}^{*} \mid M_{t}, M_{t-1}, X_{t-1}^{*}}$. Therefore, the density of interest $f_{Y_{t}, M_{t}, X_{t}^{*} \mid Y_{t-1}, M_{t-1}, X_{t-1}^{*}}$, i.e., $f_{W_{t}, X_{t}^{*} \mid W_{t-1}, X_{t-1}^{*}}$ is identified. 


\section{References}

Abbring, J., and J. Heckman (2007): "Econometric Evaluation of Social Programs, Part III: Distributional Treatment Effects, Dynamic Treatment Effects, Dynamic Discrete Choice, and General Equilibrium Policy Evaluation," in Handbook of Econometrics, Vol. $6 B$, ed. by J. Heckman, and E. Leamer, chap. 72. North-Holland.

Ackerberg, D., L. Benkard, S. Berry, and A. Pakes (2007): "Econometric Tools for Analyzing Market Outcomes," in Handbook of Econometrics, Vol. 6A, ed. by J. Heckman, and E. Leamer. North-Holland.

Aguirregabiria, V., And P. Mira (2007): "Sequential Estimation of Dynamic Discrete Games," Econometrica, 75, 1-53.

Arcidiacono, P., And R. Miller (2006): "CCP Estimation of Dynamic Discrete Choice Models with Unobserved Heterogeneity," Manuscript, Duke University.

Bajari, P., L. Benkard, And J. Levin (2007): "Estimating Dynamic Models of Imperfect Competition," Econometrica, 75, 1331-1370.

Bajari, P., V. Chernozhukov, H. Hong, and D. Nekipelov (2007): "Nonparametric and Semiparametric Analysis of a Dynamic Game Model," Manuscript, University of Minnesota.

Benkard, L. (2004): "A Dynamic Analysis of the Market for Wide-bodied Commercial Aircraft," Review of Economic Studies, 71, 581-611.

Blevins, J. (2008): "Sequential MC Methods for Estimating Dynamic Microeconomic Models," Duke University, working paper.

Cunha, F., J. Heckman, and S. Schennach (2006): "Estimating the Technology of Cognitive and Noncognitive Skill Formation," manuscript,University of Chicago.

Doraszelski, U., And A. PAKes (2007): "A Framework for Dynamic Analysis in IO," in Handbook of Industrial Organization, Vol. 3, ed. by M. Armstrong, and R. Porter, chap. 30. North-Holland.

Ericson, R., And A. Pakes (1995): "Markov-Perfect Industry Dynamics: A Framework for Empirical Work," Review of Economic Studies, 62, 53-82. 
Heckman, J., And S. Navarro (2007): "Dynamic discrete choice and dynamic treatment effects," Journal of Econometrics, 136, 341-396.

Hotz, J., And R. Miller (1993): "Conditional Choice Probabilties and the Estimation of Dynamic Models," Review of Economic Studies, 60, 497-529.

Hu, Y., And S. Schennach (2008): "Instrumental variable treatment of nonclassical measurement error models," Econometrica, 76, 195-216.

Hu, Y., And M. Shum (2008): "Nonparametric Identification of Dynamic Models with Unobserved State Variables," Jonhs Hopkins University, Dept. of Economics working paper \#543.

Magnac, T., and D. Thesmar (2002): "Identifying Dynamic Discrete Decision Processes," Econometrica, 70, 801-816.

Pakes, A., And P. McGuire (1994): "Computing Markov-Perfect Nash Equilibria: Numerical Implications of a Dynamic Differentiated Product Model," RAND Journal of Economics, 25, 555-589.

Pakes, A., M. Ostrovsky, and S. Berry (2007): "Simple Estimators for the Parameters of Discrete Dynamic Games (with Entry/Exit Examples)," RAND Journal of Economics, 37.

Pesendorfer, M., and P. Schmidt-Dengler (2007): "Asymptotic Least Squares Estimators for Dynamic Games," Review of Economic Studies, forthcoming.

Siebert, R., And C. Zulehner (2008): "The Impact of Market Demand and Innovation on Market Structure," Purdue University, working paper. 